\title{
Work Function Reduction by a Redox-Active Organometallic Sandwich Complex
}

\author{
Alexander S. Hyla ${ }^{1,2}$, Paul Winget ${ }^{1}$, Hong $\mathrm{Li}^{1,2}$, Chad Risko ${ }^{1, \neq}$, and Jean-Luc Brédas ${ }^{2, *}$ \\ ${ }^{1}$ School of Chemistry \& Biochemistry and Center for Organic Photonics and Electronics \\ Georgia Institute of Technology \\ Atlanta, Georgia 30332-0400 \\ ${ }^{2}$ Solar \& Photovoltaics Engineering Research Center \\ Division of Physical Science and Engineering \\ King Abdullah University of Science and Technology \\ Thuwal 23955-6900, Kingdom of Saudi Arabia \\ ₹ Current address: Department of Chemistry and Center for Applied Energy Research, \\ University of Kentucky, Lexington, Kentucky 40506 \\ *E-mail: jean-luc.bredas@kaust.edu.sa
}

(C) 2016. This manuscript version is made available under the Elsevier user license http://www.elsevier.com/open-access/userlicense/1.0/ 


\begin{abstract}
We have investigated, at the density functional theory level, the geometric and electronic structures of the pentamethyliridocene $\left(\operatorname{IrCpCp}{ }^{*}\right)$ monomer and dimer adsorbed on the $\mathrm{Au}(111)$ and indium tin oxide (ITO) (222) surfaces, as well as their impact on the work functions. Our calculations show that the adsorption of a monomer lowers the work function of ITO(222) by $1.2 \mathrm{eV}$ and $\mathrm{Au}(111)$ by $1.2-1.3 \mathrm{eV}$. The main origin for this reduction is the formation of an interface dipole between the monomer and the substrate via charge transfer. Dimer adsorption as well as adsorption of possible byproducts formed from dimer bond-cleavage in solution, show a lesser ability to lower the work function.
\end{abstract}




\section{Introduction}

Organic optoelectronic devices, such as organic photovoltaic devices (OPVs), organic lightemitting diodes (OLEDs), or organic thin-film transistors (OTFTs), hold promise as they may be printed or processed at low cost over large areas and on flexible substrates. ${ }^{1-4}$ One key part of the processing is the fabrication of a low work function $(\Phi)$ electrode for electron injection to (for OLEDs), or collection from (for OPVs) the electron transport levels of organic semiconductors. Traditional low- $\Phi$ metals, e.g. Ca or $\mathrm{Mg}$, are highly reactive and have to be deposited on top of the organic semiconductor layer under inert atmosphere conditions. ${ }^{5-6}$ Thus, alternative low- $\Phi$ electrodes are highly desirable.

Previous approaches include modifying an air-stable high work function transparent conducting electrode, such as indium tin oxide (ITO), by depositing a thin layer of a selected material to reduce its work function within an inverted device architecture. One of the most successful strategies is the deposition of a thin layer of a polymer containing simple aliphatic amine groups onto the conductor surfaces; for instance, Zhou et al. introduced a "universal" method for decreasing the $\Phi$ of a number of conducting electrodes based on commercially available polyethylenimine (ethoxylated) $(\mathrm{PEI}(\mathrm{E})) .^{5}$ For conducting surfaces including metals, metal oxides, polymers, and graphene, it was found that the physisorption of $\mathrm{PEI}(\mathrm{E})$ could reduce the work function by as much as $1.8 \mathrm{eV}$. Such a large work function decrease has been analyzed by density functional theory (DFT) calculations and shown to come from a joined contribution of interface and molecular dipoles aligned in a cooperative fashion. ${ }^{5}$ 
Another approach, based on a redox-active material, was taken by Osikowicz et al. Tetrakis(dimethylamino)ethylene (TDAE) was deposited on ITO and led to a $\Phi$ decrease of $0.9 \mathrm{eV}$, attributed to a double electron transfer to the ITO surface. ${ }^{7}$ TDAE was also used to decrease the $\Phi$ of gold by $1.3 \mathrm{eV}$, originating from a single electron transfer from TDAE to the gold surface. ${ }^{8}$ Bröker et al. reported a reduction of the $\Phi$ of gold by $2.2 \mathrm{eV}$ by using an air stable molecular donor layer composed of neutral methyl viologen (MV0), which underwent electron transfer with the gold surface. ${ }^{9}$ Hofmann et al. used viologen and tetrathiafulvalene (TTF) to reduce $\Phi$ of gold, silver, and copper by as much as $1.2 \mathrm{eV} .{ }^{10}$ Bröker et al. reported reduction of $\Phi$ of $\mathrm{Au}(111), \mathrm{Ag}(111)$, and $\mathrm{Cu}(111)$ by as much as $1.4 \mathrm{eV}$ by using a molecular donor layer composed of 9,9'-ethane-1,2-diylidene-bis(N-methyl-9,10-dihydroacridine) (NMA). ${ }^{11}$ Li et al. used acridine orange base $(\mathrm{AOB})$ as an n-type dopant to reduce the $\Phi$ of gold by $1.9 \mathrm{eV}{ }^{12}$ this $\Phi$ reduction is accredited to the formation of an interface dipole, caused by a single electron transfer from the AOB to the gold substrate.

Redox-active, organometallic sandwich complexes have been investigated more recently and can be stronger reductants than TDAE, MVO, or AOB. ${ }^{13-20}$ The major advantage of such compounds is that they can be applied as air- (and solution) stable dimers, and can be solution deposited. Upon reaction with an appropriate acceptor, two different mechanisms may occur: (i) either cleavage of the dimer followed by electron transfer from monomer to acceptor; or (ii) electron-transfer reaction between the dimer and the acceptor followed by dissociation of the dimer cation. ${ }^{13-15}$ The products of this decomposition reaction are shown in Figure 1. The redox activity of these compounds originates from the conversion of a ligand-centered radical with $\eta^{4}$ - 
$C p\left(\eta^{5}-C p^{*}\right) / \eta^{5}-C p\left(\eta^{4}-C p^{*}\right)$ coordination to a metal-centered radical with $\eta^{5}-C p\left(\eta^{5}-C p^{*}\right)$ coordination. With a Group 8 central atom, the metallocene complex formally carries 19 electrons, and consequently has a low ionization potential. These sandwich compounds have

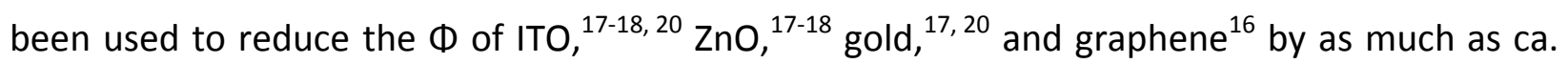
$1.9 \mathrm{eV}$.

Here, we investigate these organometallic compounds at the density functional theory (DFT) level to understand: (i) the mechanism of work function modification $(\Delta \Phi)$; and (ii) the contributions from potential synthetic byproducts. We consider the interaction of a metal ( $\mathrm{Au})$ and a metal oxide (ITO) surface with pentamethyliridocene (IrCpCp*), a prototypical 19electron sandwich complex, its dimer precursor, and potential byproducts. We have evaluated the electron donating ability of both the dimer and monomer, in terms of the $\Phi$ reduction of $\mathrm{Au}(111)$ and ITO(222) surfaces.
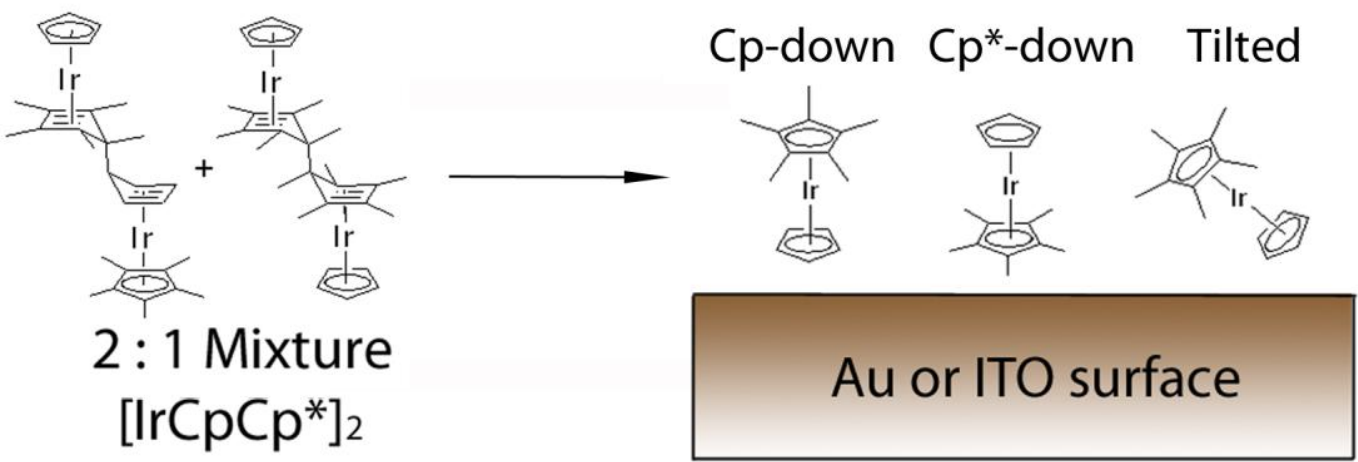

Figure 1: The IrCpCp* dimer and the possible orientations of the monomers and their abbreviations used throughout this work. 


\section{Computational Methodology}

To describe the ITO(222) surface, we use the repeated-slab approach and a surface structural model proposed in our previous work, ${ }^{21}$ which consists of 84 indium, 12 tin, 144 oxygen, and 24 hydrogen atoms in an orthogonal $24.79 \times 14.32 \AA^{2}$ surface unit cell, where all of the oxygen atoms above the top layer of metal atoms are fully saturated by hydrogen atoms. The ITO slab consists of three $\ln (\mathrm{Sn})-\mathrm{O}$ layers. When a monomer is added to the ITO surface unit cell, the resulting molecular coverage is $2.82 \times 10^{13}$ molecules $\mathrm{cm}^{-2}$. This coverage is close to the experimentally evaluated coverage based on the $\mathrm{Ir} / \mathrm{In}$ ratio obtained from the XPS experiments. $^{17}$ The atomic positions of the top layer, surface hydroxyls, and molecular adsorbate are allowed to relax over the course of geometry relaxations while the bottom two layers of the slab remain fixed.

The same repeated-slab approach is applied to the $\mathrm{Au}(111)$ surface. As the molecular coverage on gold is difficult to ascertain from experiments, ${ }^{17}$ we used three coverages, realized by orthogonal unit cells with different sizes, to explore the effects of variations in molecular coverage. The lateral dimensions of the unit cells along the [11-2] and [1-10] directions are 8.63 $\times 9.97 \AA^{2}, 17.27 \times 9.97 \AA^{2}$, and $17.27 \times 19.94 \AA^{2}$, and are taken from the experimental bulk lattice parameters. ${ }^{5,22}$ We note here that the lattice parameters optimized at the PBE-D2 level for bulk gold have been shown to be very close to the experimental values. ${ }^{23}$ When one monomer or dimer is present in the unit cell, the molecular coverages are $1.16 \times 10^{14}$ molecules $\mathrm{cm}^{-2}, 5.81 \times 10^{13}$ molecules $\mathrm{cm}^{-2}$, and $2.90 \times 10^{13}$ molecules $\mathrm{cm}^{-2}$ for the smallest, second 
largest, and largest gold unit cells, respectively. When the monomer is in the smallest unit cell and when the dimer is in the second largest unit cell, the iridium to gold ratios are the same, and these cases will be denoted as high coverage $(\mathrm{HC})$. If the monomer is in the second largest unit cell or the dimer is in the largest unit cell, the iridium to gold ratio is again the same, and these will be denoted as medium coverage (MC). The monomer in the largest unit cell will be denoted as low coverage (LC). The largest gold unit cell has approximately the same surface area as the ITO model. Each gold slab consists of five atomic layers with the bottom three layers frozen at the optimized crystal structure while the top two layers, and any molecular adsorbate, are allowed to relax over the course of geometry relaxations. In both the gold and ITO cases, the slabs are separated by a vacuum space larger than $20 \AA$.

All calculations were carried out using the Vienna Ab Initio Simulation Package (VASP). ${ }^{24-25}$ The calculations were performed with plane-wave basis sets with an energy cut off of $400 \mathrm{eV}$ and $300 \mathrm{eV}$ for the gold and ITO calculations, respectively; the projector augmented wave (PAW) method $^{26}$ was used to describe the valence-core electron interactions. We chose the generalized gradient approximation (GGA) exchange-correlation functional of Perdew, Burke, and Ernzerhof $(\mathrm{PBE}),{ }^{27-28}$ augmented by the empirical D2 dispersion correction of Grimme ${ }^{29}$ in order to describe the non-specific interactions of pentamethyliridocene ${ }^{17}$ with the metal or metal oxide surfaces. While PBE-D2 has deficiencies in the binding energies and geometries, ${ }^{30}$ it gives a qualitative description, compared to more expensive methods. As the monomer has an odd number of electrons, spin-polarized calculations were performed for both the isolated monomer and the monomer on the surface. The spin-polarized results for the monomer-on- 
substrate complex show little change in the Fermi energy, magnetization, and density of states compared to those in the absence of spin-polarization (therefore, the results presented below are essentially from calculations without spin-polarization, with a detailed comparison given in the Supporting Information). Despite the limitations of PBE, it has been used to describe charge transfer systems, such as perylene-3,4,9,10-tetracarboxylicdiimide (PTCDI), ${ }^{31}$ perylene-3,4,9,10tetracarboxylic dianhydride (PTCDA) ${ }^{32}$ and $\mathrm{C}_{60}{ }^{33}$ on $\mathrm{ZnO}$, as well as a donor-acceptor complex of tetrafluorotetracyanoquinodimethane ( $F_{4}$-TCNQ) with t-butyl carbazole-phosphonic acid modified ITO. $^{34}$ In these earlier works, the work function changes and charge-transfer characters have been calculated at the PBE level and good agreement with experiment has been reported. ${ }^{31-33}$ In the gold calculations, a $2 \times 2 \times 1$ Monkhorst-Pack k-point grids was used for geometry optimizations for all unit cells, while $6 \times 6 \times 1,3 \times 6 \times 1$, and $3 \times 3 \times 1$ MonkhorstPack k-point grids were used for self-consistent total-energy calculations for the smallest, second largest, and the largest unit cells, respectively. In the ITO calculations, only the Г-point was employed for geometry optimizations, while a $2 \times 2 \times 1$ Monkhorst-Pack k-point grid was considered for total-energy calculations. The Methfessel and Paxton ${ }^{35}$ occupation scheme with a smearing of $0.1 \mathrm{eV}$ was used for Brillouin-zone integrations in the calculations on both gold and ITO surfaces.

To calculate the electronic structures of the isolated monomers and dimers, a periodically repeated box of $40 \times 40 \times 40 \AA^{3}$ and $45 \times 45 \times 45 \AA^{3}$ was taken with the monomer or dimer at the center of the boxes. For the isolated monomer (carrying an odd number of electrons), we compared the density of states (DOS) and Bader charges calculated ${ }^{36}$ with and without spin 
polarizations. Although there is a half-occupied molecular orbital crossing the Fermi level in the DOS for the case without spin polarizations (compare the DOS in Figures S3 and S4 in the Supporting Information, calculated for an isolated monomer with and without spinpolarizations), the Bader charges for the monomer with and without spin-polarizations are remarkably similar, with differences on the order of $0.01|e|$ (see Table S6 in the Supporting Information; note that the Gaussian smearing method was used for the isolated systems and only the Г-point was employed in such calculations). Bader charges are used here, and while it is difficult to assess the accuracy of atomic partial charges, these charges have an estimated error less than $15 \% .{ }^{37}$

Geometry optimizations were performed using a damped molecular dynamics scheme until the forces were $<0.03 \mathrm{eV} / \AA \AA \AA$ for gold calculations ${ }^{38}$ and $<0.04 \mathrm{eV} / \AA \AA$ for ITO calculations. ${ }^{39}$ To compensate for possible dipole-dipole interactions between the asymmetric slabs, a dipole sheet is inserted into the vacuum gap.

\section{Results and Discussion}

\section{Binding Configurations and Energies}

Starting from optimized structures of the bare surfaces and monomers, each of the three possible monomer configurations, shown in Figure 2, were generated with an initial monomer-

surface distance of approximately $2.5 \AA$. Two structures have the asymmetric ends of the 
sandwich complex facing the surface, with either the $\mathrm{Cp}$ or $\mathrm{Cp}^{*}$ ring co-facial to the surface, denoted as "Cp-down" or "Cp*-down". There occurs a third configuration in which the iridium interacts directly with the surface, denoted as "Tilted". The $\mathrm{Cp}^{*}$-down configuration is most stable for the monomer; we used this configuration as the starting point for the dimer optimization.

Experimentally, Au or ITO were treated in a nitrogen atmosphere with solutions of $(\operatorname{IrCpCp} *)_{2}$ in toluene. Physisorption of pentamethyliridocene is thought to be non-specific. ${ }^{17}$ Since van der Waals interactions are not well described by standard density functionals, we have included a dispersion correction developed by Grimme. ${ }^{29}$ Figure 2 shows the optimized structure and relative energies of the monomers on $\mathrm{Au}(111)$ for medium coverage (MC) (a-c) and on ITO(222) (e-g). The $\mathrm{Cp}^{*}$-down configuration is the global minimum for both surfaces, with the $\mathrm{Cp}$-down and tilted configurations lying significantly higher in energy (by nearly $3 \mathrm{eV}$ ) upon complexation to the Au surface. For the high coverage $(\mathrm{HC})$ gold surface, the global minimum is the tilted configuration (see Table S1). This is likely due to the increased intermolecular dispersion interactions at this coverage, rather than interactions with the surface. 


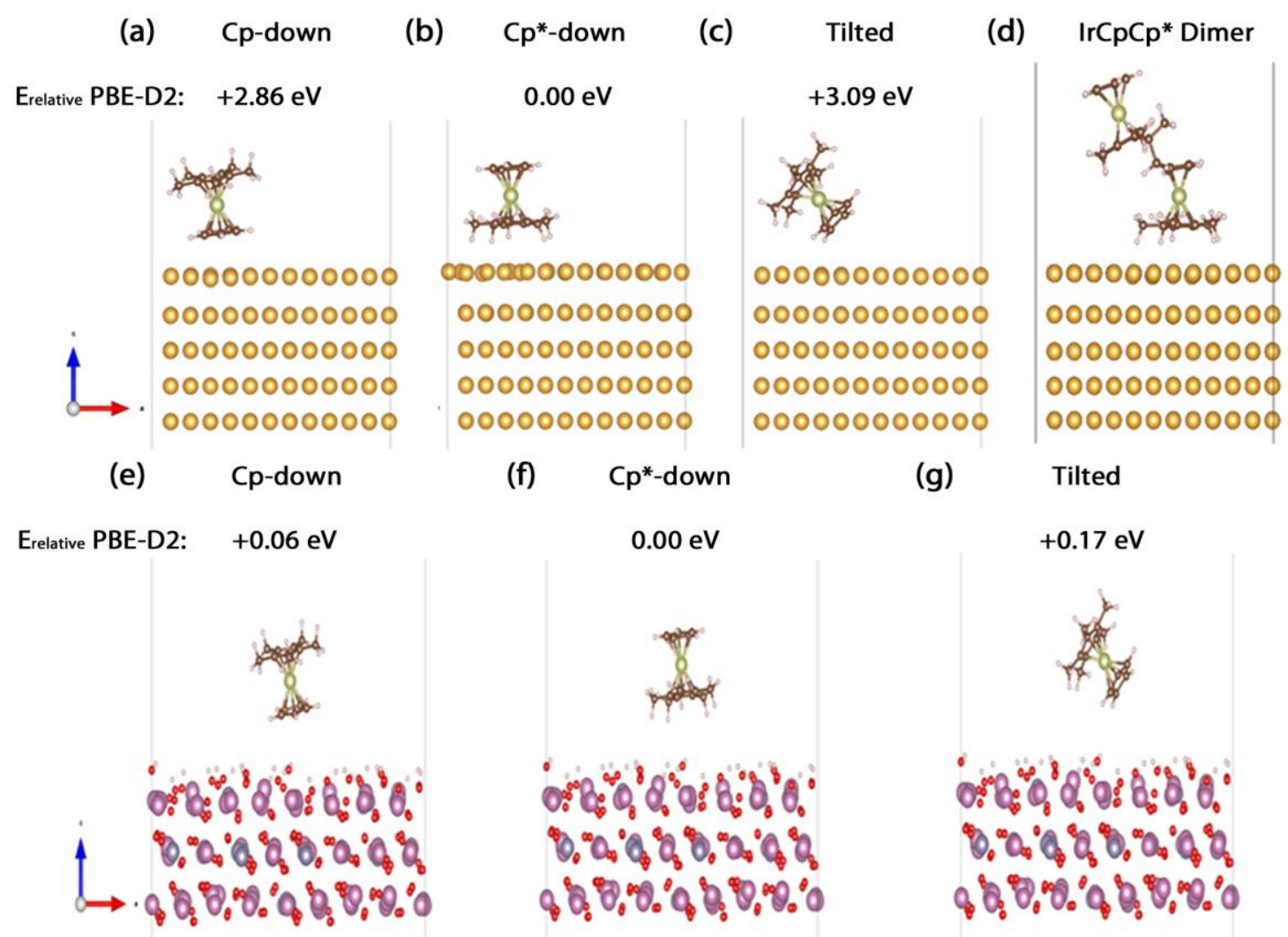

Figure 2. Optimized PBE-D2 geometries of the iridium monomers (a-c) and dimer (d) on the Au(111) surface at medium coverage and (e-g) monomers on the ITO(222) surface. The relative energies for the monomer complexes on the surfaces are also given.

The $\mathrm{Cp}$-down configuration is consistently higher in energy than the $\mathrm{Cp}^{*}$-down configuration, although the 5-membered ring is closer to the gold and ITO surfaces in the former configuration. For instance, on the MC-gold surface, the center of the $\mathrm{Cp}$ ring is $2.88 \AA$ from the surface, while the $\mathrm{Cp}^{*}$ ring is $3.19 \AA$ away (Ir atom: 4.69 and $5.00 \AA$ away, respectively) from the surface. On ITO, passivation of the surface by hydroxylation prevents as close of an approach, with the $\mathrm{Cp}$ ring $3.27 \AA$ from the surface, and the $\mathrm{Cp} *$ ring $4.09 \AA$ away (Ir atom: 5.08 and $5.91 \AA$ away, respectively). Such an energy stabilization over the $\mathrm{Cp}^{*}$-down configuration is due to the 
much stronger van der Waals interactions between the surfaces and the $\mathrm{Cp}^{*}$ ring than the $\mathrm{Cp}$ ring.

The geometric structures of the isolated monomer, dimer, and associated cations have been calculated in an earlier work, using the M06 meta-GGA exchange-correlation functional and compared to available X-ray data. ${ }^{15}$ Here we briefly describe the main geometric features obtained using a similar methodology as the surface calculations (with Gaussian smearing rather than the Methfessel and Paxton occupation scheme). The dimer contains two moieties, each of which being a $\eta^{4}-C p\left(\eta^{5}-C p^{*}\right)$ sandwich compound. The $\eta^{4}-C p$ ligand acts as a cyclopentadiene fragment, with the average Ir- $C_{\text {diene }}$ atomic distance of $2.15 \AA$ being smaller than the average $\mathrm{Ir}-\mathrm{C}_{\mathrm{Cp}^{*}}$ distance of $2.27 \AA$. . The uncoordinated $\mathrm{sp}^{3}$ carbon atom lies out of plane of the butadiene fragment, with a long Ir-C bond with an average distance of $2.78 \AA$. The $19-$ electron monomer cation exists as a sandwich compound with a small bite angle and the $\mathrm{Cp}$ and $\mathrm{Cp}^{*}$ atoms nearly equidistant, with average distances of $2.31 \AA$ and $2.34 \AA$. A schematic of these distances are shown in Figure 3.

Upon monomer binding to the MC-Au(111) surface, there is a large change in the atomic distances. The $I r-C_{C^{*}}$ distance becomes $2.19 \AA$, with the carbon atoms in the former $\eta^{4}$ cyclopentadiene ligand becoming planar, with an average C-Ir distance of $2.21 \AA$ A. The hapticity and bond distances are similar to those seen in the cation monomer structure. The dimer also undergoes geometrical changes upon interaction with gold, with the geometric change different in each monomer moiety. The monomer moiety closest to the surface experiences a 
similar evolution as the isolated monomer on the surface, with the $\mathrm{C}_{\mathrm{Cp} \text {-Ir }}$ distance contracting by $0.06 \AA$ compared to the isolated dimer, to become $2.20 \AA$. . The $\mathrm{sp}^{3}$ carbon of the monomer moiety closest to the surface remains out of plane relative to the diene fragment, although the distance to the Ir atom becomes smaller, going from 2.77 to $2.63 \AA$. The monomer moieties farther from the surface have only small changes in the bond distances. The central C-C bond expands slightly from 1.58 to $1.59 \AA$ A Details on the specific geometries of the complexes and changes after interaction with the surface are given in the Supporting Information. 

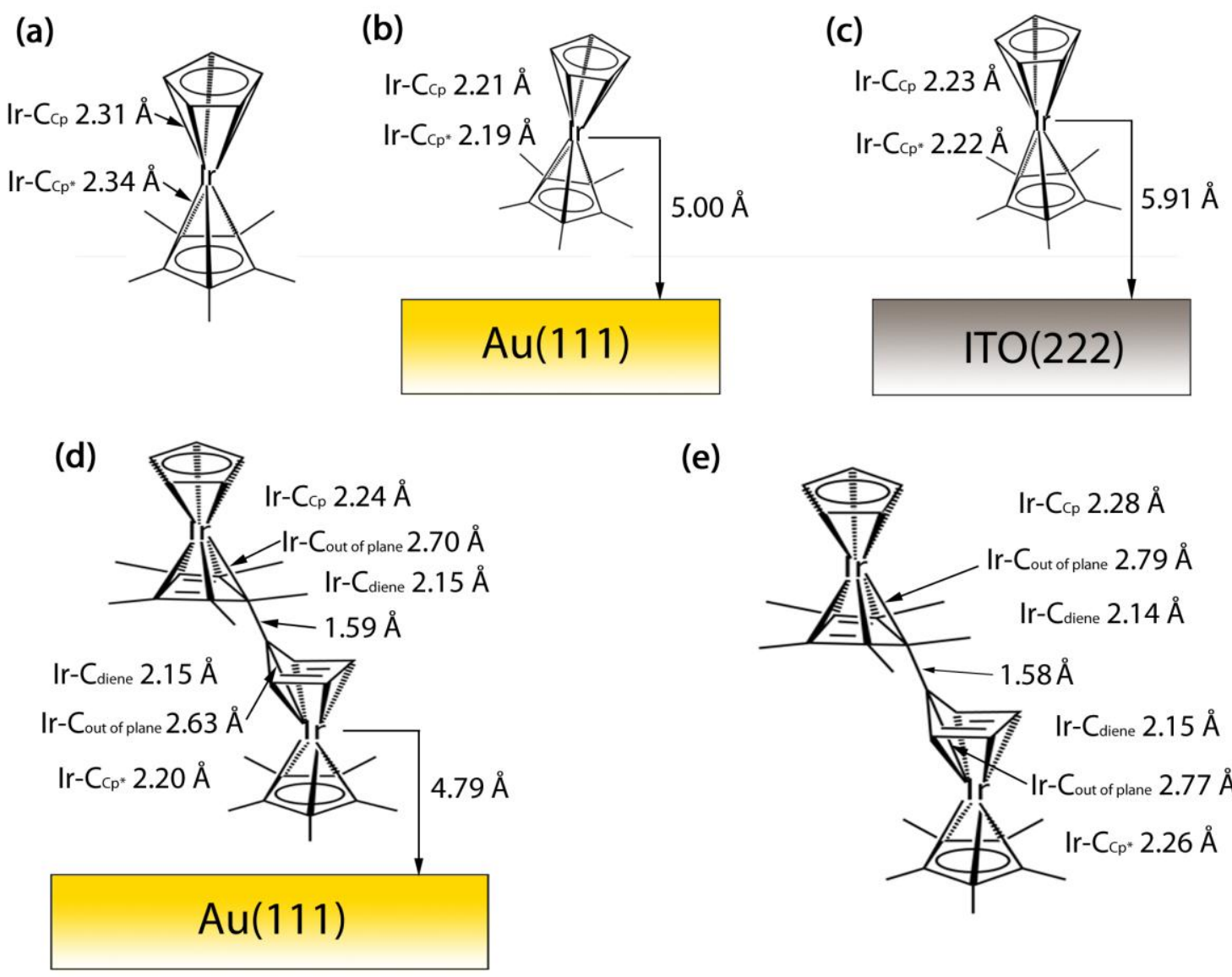

(e)

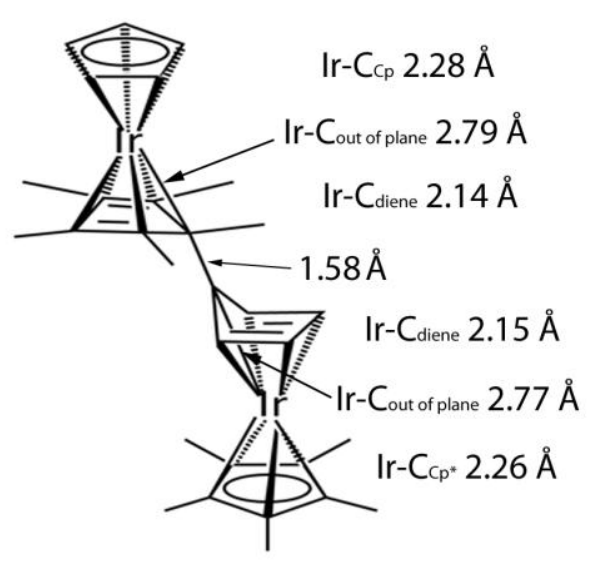

Figure 3: Average distances from the iridium to the carbon atoms of the $\mathrm{Cp}\left(\mathrm{Ir}-\mathrm{C}_{\mathrm{Cp}}\right)$ and $\mathrm{Cp}$ * rings (Ir- $\left.\mathrm{C}_{\mathrm{Cp}^{*}}\right)$ and distance to the surface (if present) in the isolated monomer (a), the $M C \mathrm{Cp}^{*}$-down monomer on gold (b), the Cp*-down monomer on ITO (c), the MC dimer on gold (d), and the isolated dimer (e). The dimer also has $\mathrm{Ir}-\mathrm{C}_{\text {diene }}$ and $\mathrm{Ir}-\mathrm{C}_{\text {out of plane }}$ distances for the rings that bind the two monomer portions together.

\section{Work function modification by monomer}

The work function $(\Phi)$ of a bare or modified surface is calculated using the definition:

$$
\Phi=\mathrm{V}_{\text {vac. }}-\mathrm{E}_{\mathrm{F}}
$$


where $\mathrm{V}_{\mathrm{vac}}$. is the plane averaged electrostatic potential energy of an electron in the vacuum region away from the slab, at a distance far enough away that the potential energy has reached its asymptotic value; $E_{F}$ is the Fermi energy of the system. For the $A u(111)$ surface, the calculated value is $5.14 \mathrm{eV}$, which compares very well to experimental data of $4.93-5.22 \mathrm{eV}^{12}$, 17,40 and previous calculations. ${ }^{38,40-42}$ The work function of the completely hydroxylated ITO surface considered here is $3.03 \mathrm{eV}$, which is substantially smaller than the experimental results that are in the range $4.0-6.1 \mathrm{eV}$. We note that the experimental value is sensitive to preparation conditions and nature of measurements; ${ }^{17,43-45}$ also, when the surface $\mathrm{OH}$ coverage is reduced to $1 / 6$ of the current value $\left(6.76 \times 10^{14} / \mathrm{cm}^{2}\right)$, the calculated work function of the model ITO surface reaches $4.24 \mathrm{eV} .{ }^{46}$ We have shown earlier that, in spite of the low value of the calculated $\Phi$ for this ITO model surface, the work function modifications upon deposition of various monolayers on the surface, were reproduced accurately. ${ }^{47}$

The average work function modification, $\Delta \Phi$, for the three $\operatorname{IrCpCp} *$ monomers on MC-gold and ITO, and the Cp*-down monomer on LC-gold are presented in Table 1. Results for all of the coverages can be found in Table S3 in the Supporting Information. The average modification for the $\mathrm{Au}(111)$ by $\mathrm{MC}$-monolayer is $-2.64 \mathrm{eV}$, with values ranging from -2.39 to $-2.80 \mathrm{eV}$ for the different configurations. The calculated $\Delta \Phi$ at this coverage is overestimated compared to the experimental value of $-1.87 \mathrm{eV}$. It is important to note, however, that the calculated work function is strongly coverage dependent; for example, the $\mathrm{Cp}^{*}$-down configuration at $\mathrm{LC}$ is calculated to have a work function modification of $-1.32 \mathrm{eV}$, while at $M C$ and $\mathrm{HC}$, the work function modifications are $-2.39 \mathrm{eV}$ and $-3.44 \mathrm{eV}$, respectively. Since the coverage on the 
$\mathrm{Au}(111)$ has not been determined experimentally, the discrepancy can (at least partly) be attributed to variations in the coverages between theory and experiment. The modification due to a monolayer of the monomer on ITO of $-1.23 \mathrm{eV}$ is similar to the modification seen experimentally, $-1.34 \mathrm{eV}$; in this case, the surface coverage considered in the calculations is in fact close to the experimental coverage. ${ }^{17}$

A decomposition scheme, discussed extensively in our earlier work, ${ }^{39,48}$ can be used to gain a better understanding of the factors leading to the total work function change. The $\Delta \Phi$ of the surfaces due to the adsorption of a molecular layer can be decomposed into three terms:

$$
\Delta \Phi=\Delta \mathrm{V}_{\text {int.dip. }}+\Delta \mathrm{V}_{\text {mol. }}+\Delta \mathrm{V}_{\text {geom }}
$$

where $\Delta \mathrm{V}_{\text {int.dip. }}$ is the contribution of the dipole formed at the very interface between molecular layer and surface; $\Delta \mathrm{V}_{\text {mol }}$. is the electrostatic potential energy change across an isolated molecular layer in vacuum; and $\Delta \mathrm{V}_{\text {geom. }}$ is the work function change of the bare surface due to geometric relaxations when interacting with a layer of IrCpCp* monomers or dimers. All these terms are collected in Table 1 . The $\Delta \mathrm{V}_{\text {mol. }}$ component, a function of the dipole moment of the molecular layer perpendicular to the surface, ${ }^{49-50}$ is small in all cases as there is only a little nonzero dipole moment in pentamethyliridocene due to the slightly different electron affinities of the $\mathrm{Cp}$ and $\mathrm{Cp} \mathrm{p}^{*}$ rings. Thus, the dipole moments of the $\mathrm{Cp}$ - and $\mathrm{Cp}$ *-down configurations at $\mathrm{MC}$

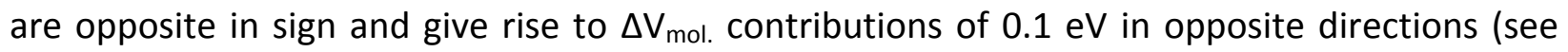
Table S3). The $\Delta \mathrm{V}_{\text {mol. }}$ contribution is reduced in the tilted configuration, as the dipole moment component perpendicular to the surface is small due to the molecular orientation. The work function change of the bare $\mathrm{Au}(111)$ surface due to geometric relaxations is limited, 
approximately $+0.1 \mathrm{eV}$. In comparison to metal surfaces, metal oxide surfaces undergo a more significant relaxation of the surface geometry upon molecular sorption. For the monomers on ITO, there is a modification of $+0.4 \mathrm{eV}$. This is consistent with previous results of chemisorbed modifiers, e.g. phosphonic acids on $\mathrm{ITO}^{39}$ vs. thiols on gold. ${ }^{42}$

The largest component of the overall work function modification is the $\Delta \mathrm{V}_{\text {int.dip. }}$ term, associated with charge transfer, the "cushion" effect, and/or redistribution at the interface. This contribution is illustrated in Figure 4 for the $\mathrm{Cp}^{*}$-down configuration at $\mathrm{MC}$ on the $\mathrm{Au}(111)$ surface. There is a large charge redistribution upon bonding, with electron transfer from the monolayer to the metal (see Figure $4 \mathrm{~b}$ for electron density decrease at the monolayer located at $\sim 20 \AA$ from the bottom of the unit cell and electron density increase in the metal at $<\sim 18 \AA$ ). This is reflected in the sum of the Bader charges, for instance, for the $\mathrm{Cp}^{*}$-down complex when interacting with the MC-gold surface. In this case, $0.42|\mathrm{e}|$ is transferred from the $\mathrm{Cp}^{*}$-down complex to the surface (see Table 2). Interestingly, at this configuration, the Bader charge analysis shows that the electron-loss on the $\mathrm{Cp}^{*}$-ring near the surface is only $0.08|\mathrm{e}|$; this is only one third of the amount of electron-loss on the Cp-ring $(0.21|\mathrm{e}|)$ that is farther away from the surface. A similar trend is observed for the Cp-down configuration at MC (see Table 2), where the electron-loss on the $\mathrm{Cp}$-ring (which is closer to the surface than $\mathrm{Cp}^{*}$ ) is $0.1|\mathrm{e}|$ lower than on the $\mathrm{Cp}^{*}$-ring. Such a trend could potentially come from a back-donation of electrons from the gold surface to the monomer complex. 
As for the work function modification, there exists a coverage dependence of the charge rearrangement, as the sum of the Bader charges shows that the $\mathrm{Cp}^{*}$-down configuration at $\mathrm{HC}$ has a charge transfer of $0.38|\mathrm{e}|$ and at LC, $0.52|\mathrm{e}|$. When the sums of Bader charges are divided by the surface area of the respective unit cells, the $\mathrm{Cp}^{*}$-down configuration has charge transfer of $4.38 \times 10^{-3}|\mathrm{e}| / \AA^{2}$ at HC, $2.44 \times 10^{-3}|\mathrm{e}| / \AA^{2}$ at $M C$, and $1.51 \times 10^{-3}|\mathrm{e}| / \AA^{2}$ at LC. This shows that even though there is more charge being transferred per monomer to the surface in the MC and LC cases, the total charge transferred decreases per unit area.

A comparison of the work function modification with respect to packing density reflects the significant depolarization effects that occur in these systems. For the Au(111)-LC Cp*-down system, the $\Phi$ decrease is $-1.32 \mathrm{eV}$; in the $\mathrm{MC}$ system that has double the monomer density, $\Phi$ decreases by $-2.52 \mathrm{eV}$, which is less than the $-2.64 \mathrm{eV}$ value expected with no depolarization. The HC-surface has even more depolarization (calculated as $-3.44 \mathrm{eV}$ versus $-5.28 \mathrm{eV}$ as expected if no depolarization), indicating that further increases in coverage would result in lesser changes to the work function.

For the monomer complex on ITO, an average charge of $0.55 \pm 0.02|\mathrm{e}|$ is transferred. Similar to the MC $A u(111)$ case shown in Figure 4b, the overall charge transfer on ITO (see Figure S1b) is comprised of a concerted loss of charge density in both $\mathrm{Cp}$ rings as well as on the central Ir atom, ultimately leading to a large decrease in the plane-averaged electrostatic potential energy above the modified surface, shown in Figure S1c. The conclusion that the interface dipole is the main component of the calculated work function modification is supported by UPS 
measurements. ${ }^{17}$ There is only a small shift in the energy of the valence band maximum of UPS spectra observed after the monomer is added, implying that the bulk of the $\Delta \Phi$ is caused by a large shift of the vacuum level, resulting from the interface dipole formed between the monomer and the substrate. ${ }^{17}$

Table 1: Experimental and calculated work function measurements and calculated work function modification breakdown for the monomers and dimer on gold and monomers on the ITO surface. All calculations have been performed at the PBE-D2 level. Values are in eV.

\begin{tabular}{|c|c|c|c|c|c|c|}
\hline System & $\operatorname{Exp} . \Phi^{a}$ & Calc. $\Phi$ & Calc. $\Delta \Phi$ & $\Delta \mathbf{V}_{\text {int. dip. }}$ & $\Delta \mathbf{V}_{\text {geom. }}$ & $\Delta \mathbf{V}_{\text {mol. }}$ \\
\hline $\mathrm{Au}(111)$ & $5.22 \pm 0.03$ & $5.14^{b} / 5.15^{c}$ & --- & --- & --- & --- \\
\hline $\mathrm{Au}(111)+\operatorname{IrCpCp} *$ & $3.35 \pm 0.03$ & ---- & ---- & ---- & ---- & ---- \\
\hline Avg. $A u(111)+\operatorname{IrCpCp} * M C^{d}$ & ---- & 2.62 & -2.52 & -2.55 & 0.01 & -0.03 \\
\hline $\mathrm{Au}(111)+{\text { Dimer } M C^{d}}^{d}$ & --- & 3.95 & -1.19 & -1.44 & 0.03 & 0.22 \\
\hline$A u(111)+\operatorname{Ir~Cp} *$-down $L C^{e}$ & ---- & 3.83 & -1.32 & -1.47 & 0.06 & 0.09 \\
\hline ITO(222) & $4.63 \pm 0.04$ & 3.03 & ---- & ---- & ---- & ---- \\
\hline Avg. ITO(222)+IrCpCp* & $3.29 \pm 0.09$ & 1.80 & -1.23 & -1.61 & 0.42 & -0.02 \\
\hline
\end{tabular}

${ }^{\mathrm{a}}$ Reference $17^{\mathrm{b}}$ Calculated work function for the MC bare gold surface ${ }^{\mathrm{C}}$ Calculated work function for the LC bare gold surface ${ }^{d}$ MC denotes medium coverage ${ }^{e}$ LC denotes low coverage. 
(a)

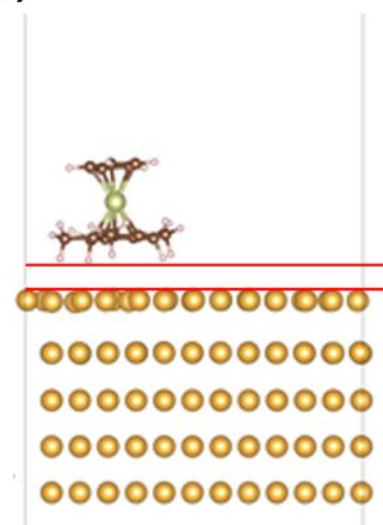

(b)

$$
\text { (b) }
$$

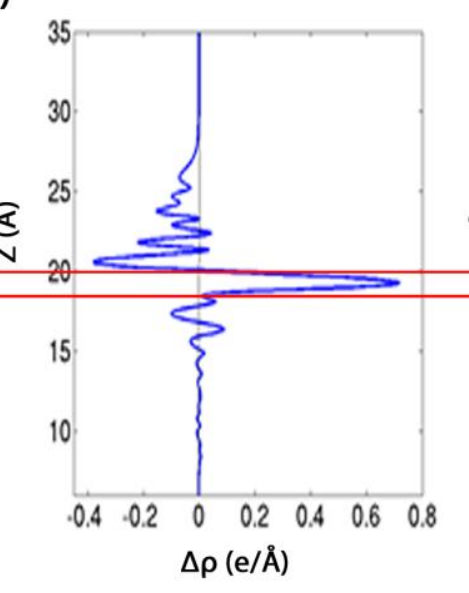

(c)

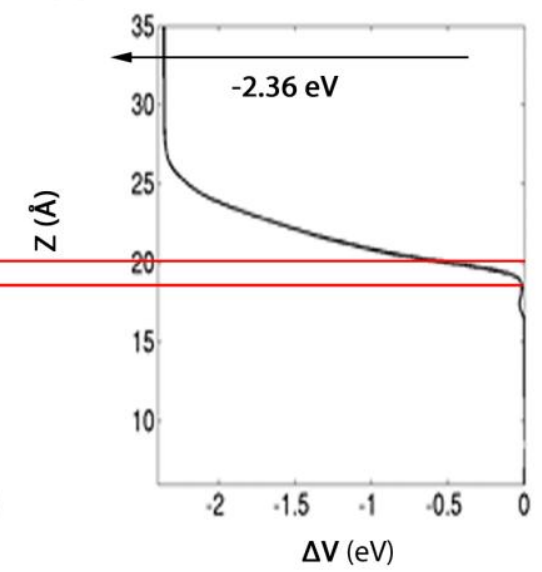

Figure 4: Representation of the $\mathrm{Cp}^{*}$-down monomer on the $\mathrm{MC} \mathrm{Au}(111)$ surface calculated at the PBED2 level (a) along with graphs of charge density difference $(\Delta \rho)$ upon $\operatorname{IrCpCp*}$ adsorption (b) and the plane-averaged electrostatic potential energy $(\Delta \mathrm{V})(\mathrm{c})$. The red lines help to designate the vacuum gap between the top of the surface and the bottom of the monomer.

Table 2: Bader charges for the components of the isolated monomer and dimer as well as the MC dimer, $\mathrm{Cp}$ *-down, $\mathrm{Cp}$-down, and hydrogen abstraction modified $\mathrm{Cp}$ *-down configurations on gold. All values in $|\mathrm{e}|$.

\begin{tabular}{lllll}
\hline System & Total & Ir & Cp ring & Cp* $^{*}$ ring \\
\hline Isolated Cp*-down & 0.00 & $0.34^{\mathrm{a}}$ & $-0.21^{\mathrm{a}}$ & $-0.13^{\mathrm{a}}$ \\
Isolated Dimer & 0.00 & $0.39^{\mathrm{b}}$ & $-0.16^{\mathrm{b}}$ & $-0.19^{\mathrm{b}}$ \\
$\mathrm{Au}(\mathbf{1 1 1})$ Ir Cp*-down MC & 0.42 & 0.47 & -0.001 & -0.05 \\
$\mathrm{Au}(\mathbf{1 1 1})$ Ir H Abstraction Cp*-down MC & 0.04 & 0.37 & -0.22 & -0.12 \\
$\mathrm{Au}(\mathbf{1 1 1})$ Ir Dimer MC & 0.19 & $0.43^{\mathrm{c}}$ & $-0.09^{\mathrm{c}}$ & $-0.11^{\mathrm{c}}$ \\
$\mathrm{Au}(\mathbf{1 1 1})$ Ir Cp-down MC & 0.59 & 0.45 & -0.01 & 0.15 \\
\hline
\end{tabular}

${ }^{a}$ Results for the spin-polarized isolated Cp*-down monomer. ${ }^{b}$ Results for the monomer portion with the $\mathrm{Cp}^{*}$ ring unbound to the other monomer portion. ${ }^{c}$ Results for the monomer portion closest to the gold surface. 


\section{Work function modification by dimer}

Table 1 demonstrates that the physisorption of monomers leads to large decreases in $\Phi$ on both gold and ITO substrates via electron transfer. We now turn to a discussion of changes in $\Phi$ that can be observed when the dimer is applied to the surface. There we considered the $\mathrm{Au}(111)$ surface. In these calculations, the dimer was placed in the largest gold unit cell (i.e., with the same iridium-to-gold ratio as the monomers in the MC cases), and the configuration of the dimer was taken to be $\mathrm{Cp}^{*}$-down as this is the most stable configuration of the monomers at the $\mathrm{MC}$ density. Adsorption of the dimer leads to a $\Delta \Phi$ of $-1.19 \mathrm{eV}$, a value more than $1.4 \mathrm{eV}$ smaller than if two monomers were deposited on the surface, but only slightly smaller than the value for a single monomer on the $\mathrm{LC}$ surface. The $\Delta \mathrm{V}_{\text {mol. }}$ component, calculated to be $0.22 \mathrm{eV}$, is almost twice the value for the $\mathrm{Cp}^{*}$-down configuration of the monomer. This results can be rationalized when considering the optimized geometry of the dimer in Figure $2 \mathrm{~d}$; the two monomer-like components of the dimer are both $\mathrm{Cp}^{*}$-down, with only a slight tilt to the component that is farthest away from the surface, which means that the individual dipole moments are nearly additive. As in the monomer case, the major component to $\Delta \Phi$ is the interface dipole. The modification of the work function due to this charge rearrangement is $1.44 \mathrm{eV}$, which is nearly identical to the low coverage monomer value of $-1.47 \mathrm{eV}$. The fragment closest to the surface has a negative charge of $0.22|\mathrm{e}|$ while the fragment further from the surface remains neutral. Both the geometric features and the charge distribution describe the dimer on the Au surface as two nearly separate monomer moieties: the moiety closest to the 
surface has undergone a change in hapticity and substantial electron transfer while the second moiety is loosely bound to the cationic moieties on the surface.

\section{Modification of the monomers}

Other molecular species are possible on the surface and can be formed when the central C-C bond in the dimer is cleaved and the 19-electron monomers can further react, for instance, via coupling with other radicals or via hydrogen- or halogen-atom abstraction. ${ }^{51}$ Here, we consider a possible reaction where the monomer has abstracted a hydrogen atom from the solvent used during the application of the dopant. In the $\mathrm{Cp}^{*}$-down configuration, this hydrogen atom enforces a $\eta^{4}-C p\left(\eta^{5}-C p^{*}\right)$ coordination, with an 18-electron $\operatorname{Ir}(I)$ monomer-like structure. In order to determine if the presence of such an $\operatorname{Ir}(\mathrm{I})$ complex can alter the work function, we carried out calculations where the $\mathrm{Cp}$ or $\mathrm{Cp}^{*}$ ring farthest from the surface is modified by adding an extra hydrogen atom. We note that this maintains the non-specific binding of the 19electron monomer, but acts to prevent electron transfer. On both surfaces, $\mathrm{Cp}$-down and Tilted binding configurations are maintained where the $\mathrm{Cp}^{*}$-down configurations are no longer stable; optimization of the systems from this latter starting point leads to a configuration that resembles the tilted configuration. The geometries of all the modified complexes are shown in Figure S2 with a representative example illustrated in Figure 5. The $\Delta \Phi$ and $\Delta \Phi$ breakdowns in terms of Eq. 2 for these modified monomers are shown in Table 3. The work function is reduced by $0.95 \mathrm{eV}$ on the $\mathrm{Au}(111)$ surface and by $0.03 \mathrm{eV}$ on ITO. Compared with the modifications from the monomers in Table 1, these are significantly smaller values. The $\Delta \Phi$ breakdown again 
shows that for the modified monomers on gold, the majority of the modification continues to be due to the interface dipole, while there is almost no change in $\Phi$ from the ITO surface. For the modified monomer on gold, the interface dipole is approximately $0.5 \mathrm{eV}$ smaller than the corresponding value for the dimer on gold. The limited magnitude of the work function modification here underlines that the electron-transfer process is the defining feature of these dopants; the presence of such modified structures on the surface cannot account for the observed $\Phi$ modification.

(a)

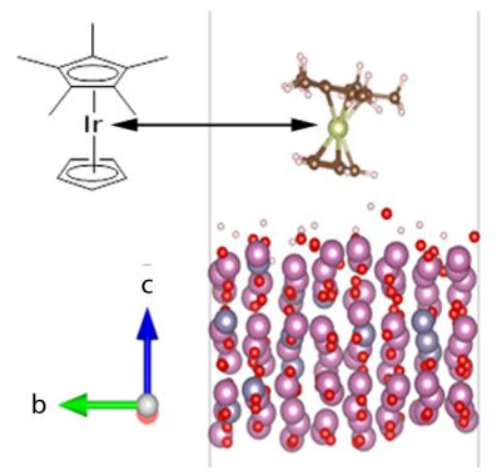

(b)

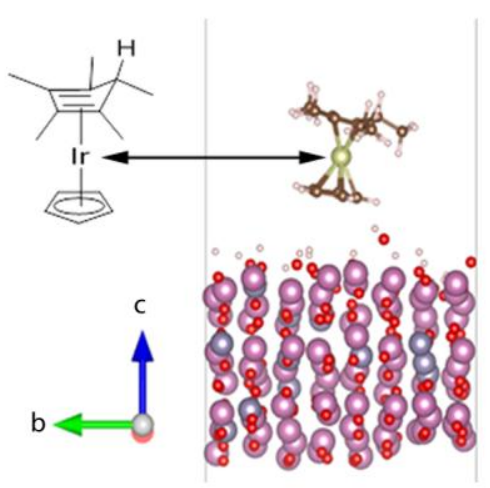

Figure 5. Comparison of the geometry of the unmodified $\mathrm{Cp}$-down monomer (a) and the modified $\mathrm{Cp}$ down monomer, with an extra hydrogen atom (b) on the ITO(222) surface. 
Table 3: Comparison of the $\Delta \Phi$ and $\Delta \Phi$ breakdowns of the modified monomers to the monomers on the gold and ITO surfaces. The monomers on gold are at the medium coverage. All values in $\mathrm{eV}$.

\begin{tabular}{l|ll|lll}
\hline System & Calc. $\Phi$ & Calc. $\Delta \Phi$ & $\Delta \mathbf{V}_{\text {int. dip. }}$ & $\Delta \mathbf{V}_{\text {geom. }}$ & $\Delta \mathbf{V}_{\text {mol. }}$ \\
\hline Au(111) & 5.14 & --- & --- & --- & --- \\
Avg. Au(111)+IrCpCp* + H & $4.19 \pm 0.11^{\mathrm{a}}$ & $-0.95 \pm 0.11^{\mathrm{a}}$ & $-0.92 \pm 0.09^{\mathrm{a}}$ & $0.06 \pm 0.006^{\mathrm{a}}$ & $-0.01 \pm 0.04^{\mathrm{a}}$ \\
ITO(222) & 3.03 & --- & -- & --- & --- \\
Avg. ITO(222)+IrCpCp* + H & $3.00 \pm 0.03^{\mathrm{a}}$ & $-0.03 \pm 0.03^{\mathrm{a}}$ & $0.01 \pm 0.01^{\mathrm{a}}$ & $0.003 \pm 0.01^{\mathrm{a}}$ & $-0.02 \pm 0.03^{\mathrm{a}}$ \\
\hline
\end{tabular}

${ }^{\text {a }}$ Average and standard deviations of the results over the three investigated configurations.

\section{Conclusions}

The major factors contributing to the large work function modifications due to IrCpCp* monomers on ITO and gold substrates have been examined using DFT. The calculated $\Phi$ reduction caused by deposition of the monomer is $\sim 1.2 \mathrm{eV}$ for the ITO(222) surface and $\sim 1.3 \mathrm{eV}$ for the $\mathrm{Au}(111)$ surface at low molecular coverage; it is mainly associated with the contribution of a large interface dipole. Our results are in agreement with the UPS measurements, ${ }^{17}$ which has attributed such work function reductions to the formation of interface dipoles. This interface dipole has a large contribution from charge transfer between the iridium complex and the surface, which only occurs when the central C-C bond of the dimer is cleaved to form the redox-active monomer species. While previous work had only hypothesized that such a charge transfer occurs, our work clearly highlights it on surfaces with different chemistries.

Calculations for the IrCpCp* dimer on the surface show smaller modifications of the surface work function as the C-C bond is only partially cleaved, which limits charge transfer to the 
surface. This partial cleavage shows that the C-C bond strength and cleavage mechanism are critical parameters determining the ability of the species to donate electrons to the surface. Adding a hydrogen atom to the $\mathrm{Cp}$ ring farther from the surface has been shown to reduce the amount of charge transfer, which leads to considerably smaller work function modifications than for the dimer or unmodified monomers.

Overall, the redox-active materials examined in this investigation represent an interesting class of surface modifiers in addition to covalently bound modifiers such as phosphonic acids, thiols, or carboxylic acids. Redox-active dopants are attractive from the standpoint of applications to a broad variety of substrates, compared to other modifiers which require specific chemical reactions to occur between the substrate surface and a binding group. The tradeoff may come in the overall stability of the surface after modification as covalently bound modifiers have been shown to be very stable on the surface. 


\section{Acknowledgements}

The authors wish to thank Dr. Stephen Barlow, Dr. Anthony Giordano, and Prof. Seth Marder for insightful discussions. This work is based on research supported in part by the Center for Interface Science: Solar-Electric Materials (CIS:SEM), an Energy Frontier Research Center funded through the U.S. Department of Energy, Office of Science, Office of Basic Energy Sciences, under Award Number DE-SC0001084. We also acknowledge generous support from King Abdullah University of Science and Technology; we thank the IT Research Computing Team and Supercomputing Laboratory at KAUST for providing computational and storage resources. The computational resources at Georgia Tech are funded in part by the CRIF Program of the NSF under Award Number CHE-0946869.

\section{Supporting Information}

Relative energies of the monomer on gold at high coverage, distances between the monomer and the surface (both the iridium and ring closest to the surface) as well as the distance of the $C p(*)$ rings from the central iridium for the monomer and the dimer, $\Delta \Phi$ breakdown results for specific configurations of the monomers at different coverages, a figure depicting the zdependence of charge transfer and interface dipole for the Cp*-down configuration on ITO, images of the optimized geometries for the medium coverage modified monomers on gold and on ITO, $\Delta \Phi$ breakdown results for specific configurations of the modified monomers, comparison of the total energies, Fermi energies, and magnetization of the high coverage $\mathrm{Cp}^{*}$ - 
down configuration following spin-polarized and non-spin-polarized calculations, comparison of

the Bader charges from calculations with and without spin polarization, and projected density

of states plots for the isolated $\mathrm{Cp}^{*}$-down monomer both with and without spin-polarization.

\section{References}

1. Friend, R. H.; Gymer, R. W.; Holmes, A. B.; Burroughes, J. H.; Marks, R. N.; Taliani, C.; Bradley, D. D. C.; Dos Santos, D. A.; Brédas, J.-L.; Löglund, M.; Salaneck, W. R. "Electroluminescence in conjugated polymers." Nature 1999397 121-128.

2. Yu, G.; Gao, J.; Hummelen, J. C.; Wudl, F.; Heeger, A. J. "Polymer Photovoltaic Cells: Enhanced Efficiencies via a Network of Internal Donor-Acceptor Heterojunctions." Science 1995, 270 (5243), 1789-1791.

3. Yan, H.; Chen, Z.; Zheng, Y.; Newman, C.; Quinn, J. R.; Dötz, F.; Kastler, M.; Facchetti, A. "A highmobility electron-transporting polymer for printed transistors." Nature 2009, 457 (7230), 679-686.

4. Khan, S.; Lorenzelli, L.; Dahiya, R. "Technologies for Printing Sensors and Electronics over Large Flexible Substrates: A Review." IEEE Sens. J. 2015, 15 (6), 3164-3185.

5. Zhou, Y.; Fuentes-Hernandez, C.; Shim, J.; Meyer, J.; Giordano, A. J.; Li, H.; Winget, P.; Papadopoulos, T.; Cheun, H.; Kim, J.; Fenoll, M.; Dindar, A.; Haske, W.; Najafabadi, E.; Khan, T. M.; Sojoudi, H.; Barlow, S.; Graham, S.; Brédas, J.-L.; Marder, S. R.; Kahn, A.; Kippelen, B. "A Universal Method to Produce Low-Work Function Electrodes for Organic Electronics." Science 2012, 336, 327-332.

6. Jørgensen, M.; Norrman, K.; Krebs, F. C. "Stability/degradation of polymer solar cells." Sol. Energy Mater. Sol. Cells 2008, 92 (7), 686-714.

7. Osikowicz, W.; Crispin, X.; Tengstedt, C.; Lindell, L.; Kugler, T.; Salaneck, W. R. "Transparent low-work function indium tin oxide electrode obtained by molecular scale interface engineering." Appl. Phys. Lett. 2004, 85, 1616-1618.

8. Lindell, L.; Unge, M.; Osikowicz, W.; Stafström, S.; Salaneck, W. R.; Crispin, X.; de Jong, M. P. "Integer charge transfer at the tetrakis(dimethylamino)ethylene/Au interface." Appl. Phys. Lett. 2008, 92, 163302(1-3).

9. Bröker, B.; Blum, R.-P.; Frisch, J.; Vollmer, A.; Hofmann, O. T.; Rieger, R.; Müllen, K.; Rabe, J. P.; Zojer, E.; Koch, N. "Gold work function reduction by $2.2 \mathrm{eV}$ with an air-stable molecular donor layer " Appl. Phys. Lett. 2008, 93, 243303(1-3).

10. Hofmann, O. T.; Rangger, G. M.; Zojer, E. "Reducing the Metal Work Function beyond Pauli Pushback: A Computational Investigation of Tetrathiafulvalene and Viologen on Coinage Metal Surfaces." J. Phys. Chem. C 2008, 112 (51), 20357-20365.

11. Bröker, B.; Blum, R.-P.; Beverina, L.; Hofmann, O. T.; Sassi, M.; Ruffo, R.; Pagani, G. A.; Heimel, G.; Vollmer, A.; Frisch, J.; Rabe, J. P.; Zojer, E.; Koch, N. "A High Molecular Weight Donor for Electron Injection Interlayers on Metal Electrodes." ChemPhysChem 2009, 10 (17), 2947-2954.

12. Li, F.; Zhou, Y.; Zhang, F.; Liu, X.; Zhan, Y.; Fahlman, M. "Tuning Work Function of Noble Metals As Promising Cathodes in Organic Electronic Devices." Chem. Mater. 2009, 21, 2798-2802.

13. Guo, S.; Kim, S. B.; Mohapatra, S. K.; Qi, Y.; Sajoto, T.; Kahn, A.; Marder, S. R.; Barlow, S. "n-Doping of Organic Electronic Materials Using Air-Stable Organometallics." Adv. Mater. 2012, 24, 699-703.

14. Guo, S.; Mohapatra, S. K.; Romanov, A.; Timofeeva, T. V.; Hardcastle, K. I.; Yesudas, K.; Risko, C.; Brédas, J.-L.; Marder, S. R.; Barlow, S. "n-Doping of Organic Electronic Materials Using Air-Stable 
Organometallics: A Mechanistic Study of Reduction by Dimeric Sandwich Compounds." Chem. - Eur. J. 2012, 18 (46), 14760-14772.

15. Mohapatra, S. K.; Fonari, A.; Risko, C.; Yesudas, K.; Moudgil, K.; Delcamp, J. H.; Timofeeva, T. V.; Brédas, J.-L.; Marder, S. R.; Barlow, S. "Dimers of Nineteen-Electron Sandwich Compounds: Crystal and Electronic Structures, and Comparison of Reducing Strengths." Chem. - Eur. J. 2014, 20, 1538515394.

16. Paniagua, S. A.; Baltazar, J.; Sojoudi, H.; Mohapatra, S. K.; Zhang, S.; Henderson, C. L.; Graham, S.; Barlow, S.; Marder, S. R. "Production of heavily $n$ - and $\mathrm{p}$-doped CVD graphene with solutionprocessed redox-active metal-organic species." Mater. Horiz. 2014, 1, 111-115.

17. Giordano, A. J.; Pulvirenti, F.; Khan, T. M.; Fuentes-Hernandez, C.; Moudgil, K.; Delcamp, J. H.; Kippelen, B.; Barlow, S.; Marder, S. R. "Organometallic Dimers: Application to Work Function Reduction of Conducting Oxides." ACS Appl. Mater. Interfaces 2015, 7 (7), 4320-4326.

18. Schlesinger, R.; Bianchi, F.; Blumstengel, S.; Christodoulou, C.; Ovsyannikov, R.; Kobin, B.; Moudgil, K.; Barlow, S.; Hecht, S.; Marder, S. R.; Henneberger, F.; Koch, N. "Efficient light emission from inorganic/organic semiconductor hybrid structures by energy level tuning." Nat. Commun. 2015, 6, 6754 (1-7).

19. Zhang, S.; Naab, B. D.; Jucov, E. V.; Parkin, S.; Evans, E. G. B.; Millhauser, G. L.; Timofeeva, T. V.; Risko, C.; Brédas, J.-L.; Bao, Z.; Barlow, S.; Marder, S. R. "n-Dopants Based on Dimers of Benzimidazoline Radicals: Structures and Mechanism of Redox Reactions." Chem. - Eur. J. 2015, 21 (30), 1087810885.

20. Akaike, K.; Nardi, M. V.; Oehzelt, M.; Frisch, J.; Opitz, A.; Christodoulou, C.; Ligorio, G.; Beyer, P.; Timpel, M.; Pis, I.; Bondino, F.; Moudgil, K.; Barlow, S.; Marder, S. R.; Koch, N. "Effective Work Function Reduction of Practical Electrodes Using an Organometallic Dimer." Adv. Funct. Mater. 2016, 26 (15), 2493-2502.

21. Paramonov, P. B.; Paniagua, S. A.; Hotchkiss, P. J.; Jones, S. C.; Armstrong, N. R.; Marder, S. R.; Brédas, J.-L. "Theoretical Characterization of the Indium Tin Oxide Surface and of Its Binding Sites for Adsorption of Phosphonic Acid Monolayers." Chem. Mater. 2008, 20, 5131-5133.

22. Owen, E. A.; Yates, E. L. "Precision measurements of crystal parameters." Philos. Mag. 1933, 15 (98), 472-488.

23. Sławińska, J.; Dabrowski, P.; Zasada, I. "Doping of graphene by a Au(111) substrate: Calculation strategy within the local density approximation and a semiempirical van der Waals approach." Phys. Rev. B 2011, 83, 245429(1-8).

24. Kresse, G.; Furthmüller, J. "Efficiency of ab-inito total energy calculations for metals and semiconductors using a plane-wave basis set." Comput. Mater. Sci. 1996, 6 (1), 15-50.

25. Kresse, G.; Furthmüller, J. "Efficient iterative schemes for ab initio total-energy calculations using a plane-wave basis set." Phys. Rev. B 1996, 54 (16), 11169-11186.

26. Blöchl, P. E. "Projector augmented-wave method." Phys. Rev. B 1994, 50 (24), 17953-17979.

27. Perdew, J. P.; Burke, K.; Ernzerhof, M. "Generalized Gradient Approximation Made Simple [Phys. Rev. Lett. 77, 3865 (1996)] Errata." Phys. Rev. Lett. 1997, 78 (7), 1396.

28. Perdew, J. P.; Burke, K.; Ernzerhof, M. "Generalized Gradient Approximation Made Simple." Phys. Rev. Lett. 1996, 77 (18), 3865-3868.

29. Grimme, S. "Semiempirical GGA-Type Density Functional Constructed with a Long-Range Dispersion Correction." J. Comput. Chem. 2006, 27, 1787-1799.

30. Ruiz, V. G.; Lu, W.; Zojer, E.; Scheffler, M.; Tkatchenko, A. "Density-Functional Theory with Screened van der Waals Interactions for the Modeling of Hybrid Inorganic-Organic Systems." Phys. Rev. Lett. 2012, 108 (14), 146103(1-5).

31. Winget, P.; Schirra, L. K.; Cornil, D.; Li, H.; Coropceanu, V.; Ndione, P. F.; Sigdel, A. K.; Ginley, D. S.; Berry, J. J.; Shim, J.; Kim, H.; Kippelen, B.; Brédas, J.-L.; Monti, O. L. A. "Defect-Driven Interfacial 
Electronic Structures at an Organic/Metal-Oxide Semiconductor Heterojunction." Adv. Mater. 2014, 26 (27), 4711-4716.

32. Gruenewald, M.; Schirra, L. K.; Winget, P.; Kozlik, M.; Ndione, P. F.; Sigdel, A. K.; Berry, J. J.; Forker, R.; Brédas, J.-L.; Fritz, T.; Monti, O. L. A. "Integer Charge Transfer and Hybridization at an Organic Semiconductor/Conductive Oxide Interface." J. Phys. Chem. C 2015, 119 (9), 4865-4873.

33. Schulz, P.; Kelly, L. L.; Winget, P.; Li, H.; Kim, H.; Ndione, P. F.; Sigdel, A. K.; Berry, J. J.; Graham, S.; Brédas, J.-L.; Kahn, A.; Monti, O. L. A. "Tailoring Electron-Transfer Barriers for Zinc Oxide/ $\mathrm{C}_{60}$ Fullerene Interfaces." Adv. Funct. Mater. 2014, 24 (46), 7381-7389.

34. Li, H.; Winget, P.; Brédas, J.-L. "Surface Modification of Indium-Tin-Oxide Via Self-Assembly of a Donor-Acceptor Complex: A Density Functional Theory Study." Adv. Mater. 2012, 24, 687-693.

35. Methfessel, M.; Paxton, A. T. "High-precision sampling for Brillouin-zone integration in metals." Phys. Rev. B 1989, 40 (6), 3616-3621.

36. Tang, W.; Sanville, E.; Henkelman, G. "A grid-based Bader analysis algorithm without lattice bias." J. Phys.: Condens. Matter 2009, 21, 084204(1-7).

37. Jacquemin, D.; Tanqui;, L. B.; Adamo, C.; Ciofini, I. "What is the "best" atomic charge model to describe through-space charge-transfer excitations?" Phys. Chem. Chem. Phys. 2012, 14 (16), 53835388.

38. Li, H.; Duan, Y.; Coropceanu, V.; Brédas, J.-L. "Electronic structure of the pentacene-gold interface: A density-functional theory study." Org. Electron. 2009, 10, 1571-1578.

39. Li, H.; Paramonov, P. B.; Brédas, J.-L. "Theoretical study of the surface modification of indium tin oxide with trifluorophenyl phosphonic acid molecules: impact of coverage density and binding geometry." J. Mater. Chem. 2010, 20, 2630-2637.

40. Fenwick, O.; Van Dyck, C.; Murugavel, K.; Cornil, D.; Reinders, F.; Haar, S.; Mayor, M.; Cornil, J.; Samorì, P. "Modulating the charge injection in organic field-effect transistors: fluorinated oligophenyl self-assembled monolayers for high work function electrodes." J. Mater. Chem. C 2015, 3 (13), 3007-3015.

41. Li, H.; Duan, Y.; Paramonov, P. B.; Coropceanu, V.; Brédas, J.-L. "Electronic structure of selfassembled (fluoro)methylthiol monolayers on the $\mathrm{Au}(111)$ surface: Impact of fluorination and coverage density." J. Electron Spectrosc. Relat. Phenom. 2009, 174, 70-77.

42. Cornil, D.; Cornil, J. "Work-function modification of the (111) gold surface upon deposition of selfassembled monolayers based on alkanethiol derivatives." J. Electron Spectrosc. Relat. Phenom. 2013, 189, 32-38.

43. Zhou, Y.; Shim, J. W.; Fuentes-Hernandez, C.; Sharma, A.; Knauer, K. A.; Giordano, A. J.; Marder, S. R.; Kippelen, B. "Direct correlation between work function of indium-tin-oxide electrodes and solar cell performance influenced by ultraviolet irradiation and air exposure." Phys. Chem. Chem. Phys. 2012, $14,12014-12021$.

44. Sugiyama, K.; Ishii, H.; Ouchi, Y.; Seki, K. "Dependence of indium-tin-oxide work function on surface cleaning method as studied by ultraviolet and x-ray photoemission spectroscopies." J. Appl. Phys. 2000, 87 (1), 295-298.

45. Helander, M. G.; Wang, Z. B.; Qiu, J.; Greiner, M. T.; Puzzo, D. P.; Liu, Z. W.; Lu, Z. H. "Chlorinated Indium Tin Oxide Electrodes with High Work Function for Organic Device Compatibility." Science 2011, 332, 944-947.

46. Li, H.; Winget, P.; Brédas, J.-L. "Transparent Conducting Oxides of Relevance to Organic Electronics: Electronic Structures of Their Interfaces with Organic Layers." Chem. Mater. 2014, 26 (1), 631-646.

47. Hotchkiss, P. J.; Li, H.; Paramonov, P. B.; Paniagua, S. A.; Jones, S. C.; Armstrong, N. R.; Brédas, J.-L.; Marder, S. R. "Modification of the Surface Properties of Indium Tin Oxide with Benzylphosphonic Acids: A Joint Experimental and Theoretical Study." Adv. Mater. 2009, 21, 4496-4501. 
48. Wood, C.; Li, H.; Winget, P.; Brédas, J.-L. "Binding Modes of Fluorinated Benzylphosphonic Acids on the Polar ZnO Surface and Impact on Work Function." J. Phys. Chem. C 2012, 116, 19125-19133.

49. Heimel, G.; Romaner, L.; Zojer, E.; Brédas, J.-L. "Toward Control of the Metal-Organic Interfacial Electronic Structure in Molecular Electronics: A First-Principles Study on Self-Assembled Monolayers of Pi-Conjugated Molecules on Noble Metals." Nano Lett. 2007, 7 (4), 932-940.

50. Heimel, G.; Romaner, L.; Zojer, E.; Brédas, J.-L. "The Interface Energetics of Self-Assembled Monolayers on Metals." Acc. Chem. Res. 2008, 41 (6), 721-729.

51. Astruc, D. "Nineteen-Electron Complexes and Their Role in Organometallic Mechanisms " Chem. Rev. $1988,88,1189-1216$. 